\title{
Three Dimensional Relationships of Emotional Intelligence, Exercise and Stress in Adolescents
}

\author{
Soma Das ${ }^{1}$, Kutubuddin Halder ${ }^{2}$, Amit Bandyopadhyay ${ }^{1}$ \\ ${ }^{1}$ Sports and Exercise Physiology Laboratory, Department of Physiology, University of Calcutta, University College of Science and \\ Technology, Kolkata, India \\ ${ }^{2}$ Department of Education, University of Calcutta, Kolkata, India
}

Email address:

bamit74@yahoo.co.in (A. Bandyopadhyay)

\section{To cite this article:}

Soma Das, Kutubuddin Halder, Amit Bandyopadhyay. Three Dimensional Relationships of Emotional Intelligence, Exercise and Stress in Adolescents. Advances in Applied Physiology. Vol. 1, No. 1, 2016, pp. 1-7. doi: 10.11648/j.aap.20160101.11

\begin{abstract}
The present review was an attempt to accumulate the existing knowledge on the psychophysiological response to stress in adolescence. The current study is also focused to explore the effects of regular exercise and emotional intelligence on coping stress in adolescents. Neuro-physiological mechanism involved in exercise induced process of combating stress have been surveyed in addition to the psychophysiological way of emotional intelligence in managing stress in adolescents. The works on gender variation in stress response and coping strategy have also been investigated with the target age group. The available literature suggests constructive neuro-physiological role of exercise on significant reduction in stress among adolescents. Researches indicated that participation in emotional intelligence (EI) development programme and regular exercise training are potentially effective skills in overcoming stress mediated turmoil in adolescents' personal and community life. The present update strongly recommends the inclusion of regular exercise programme and practice of emotional intelligence skill in main stream school curriculum as compulsory activity for students in adolescence stage for better balanced and healthy life style in future course of time.
\end{abstract}

Keywords: Exercise, Emotional Intelligence, Stress Coping, Adolescents

\section{Introduction}

Adolescence is transitional period from childhood to adulthood. This takes place in two ways. One is physiological change which has physical and external manifestations including the rapid development, sexual maturity. The other one is psychological change (discovering one's real self and defining personal value) which is intrinsic in nature [1].

Adolescence is characterised by the development of occupational, educational and personal contexts [2] with drastic modification of individual social expectations.

Adolescents are more prone to stress induced physiological, psychological and social complications [3].This has become enormously popular field of research interest among the scientists and they have tried to locate the potent stressors and their psychophysiological effects in human health [4]. Currently, the strategies are being implemented to cope stress in two broadly classified dimensions - physiological coping and psychological coping [5]. Exercise plays a pivotal role in physiological coping of stress whereas [6], emotional intelligence has been reported to play the key role in psychological coping of stress [2].

The present review is aimed to explore the association of emotional intelligence and exercise on coping stress in adolescents and to address the existing knowledge about the psychophysiological basis of such relationship.

\section{Physiological Backdrop of Stress in the Context of Adolescent Population}

The physiological concept of stress was first introduced by Hans Selye long back in the year 1956 who defined stress as a perturbation of the body's homeostasis [7]. It may happen due to an external event or an internal drive which disturbs the normal functioning of an individual, resulting in impaired health (physiological effect) or altered behaviour (psychological effect) [8]. This demand on body and mind occurs very often in adolescents who try to cope with incessant changes in life [9]. It is caused by a multitude of demands (stressors), such as an inadequate fit between what we need and what we are capable of, and what our environment offers or permits [9]. 
It was revealed that the psychological effect of stress as manifested by altered behaviour is determined by the physiological changes at neuronal level in brain [10]. Hence the neurophysiology of stress has been drawing attention of many researchers to address the bodily effect of stress conjointly from physiological as well as psychological point of view. In this connection the effects of physiological and psychological factors on brain functioning and body aging across the life span was carried out in animal models, as well as epidemiological studies were conducted in human populations [11]. It has been reported that the onset of stress enhances the growth of new brain cells which are responsible for improved memory [12]. However, this effect is seen only when stress is intermittent. If the stress continues beyond a few moments into a prolonged period, the effect gets reversed i.e., it suppresses the ability of brain to develop new cells [13] and that in turn affects the normal neurophysiological functioning of the nervous system.

\section{The Effect of Exercise on Development of Brain Functioning}

The effect of exercise on development of brain functioning or in other words the neurophysiological effect of exercise is one of the areas of interest of the current study. Research revealed two types of effects of physical exercise on brain have been postulated, one is the acute exercise mechanism (AEM) and the other is chronic exercise mechanism (CEM) [14].

\subsection{Mechanism of Effects of Acute Exercise (AEM) on Development of Brain Functioning}

The AEM suggests that physical exercise promotes changes in the human brain by increase in metabolism, oxygenation and blood flow in the brain cells. Unfortunately as of now, limited works were conducted with human brain and most of these observations were performed in animal model $[15,16]$. Such studies on animal models confirmed that exercise modulates the major neurotransmitters of central nervous system (CNS), i.e., norepinephrine, dopamine, and serotonin that are associated with an individual's state of alertness, the pleasure and reward system and the level of anxiety, respectively. Moreover, changes in the levels of these neurotransmitters may have varied effects depending on the type(s) of receptors and the cortical areas that are activated by exercise [17]. There are some neurochemical factors like, opioids and endocannabinoids which might have released during exercise and could promote a sense of euphoria and well-being, anxiolytic effects, sedation and decreased sensitivity to pain in humans [18].

Other neuromodulators called, trophic factors are also activated by acute exercise regimen [19]. Expression levels of brain-derived neurotrophic factor (BDNF), insulin-like growth factor (IGF-1), vascular endothelial growth factor (VEGF), neurotrophin-3 (NT3), fibroblast growth factor (FGF-2), glial cell line-derived neurotrophic factor (GDNF), epidermal growth factor (EGF) and nerve growth factor (NGF) appeared to increase in conjunction with exercise interventions and those factors acted as survival, proliferation and maturation factors in specific brain cells [20]. Mechanism of action of these neurotrophic factors have also been clearly hypothesised. Upon binding to their specific receptors, they lead to the activation of Ras-GTPases and Raf-kinases proteins mediated signalling pathways, P13K (protein 13 kinase)/Akt (protein kinase B) and cAMP response element-binding (CREB) protein [20]. The aforementioned neurotrophic factors are even capable of inhibiting signals in the caspase-mediated apoptotic pathway [21]. In the CNS, these trophic factors at one way act as modulators and on the other way they get modulated by neurotransmitters, which in turn play a substantial role in determining an individual's level of cognition and behaviours. Even secretion of sex hormones was reported to be regulated by trophic factors [21].

\subsection{Mechanism of Effects of Chronic Exercise on Development of Brain Functioning}

The chronic exercise mechanism is reported to result in long-term effects on different responses and adaptations to stress than those observed during acute exercise participation (after only one session). Responses include increase in cerebral blood flow (CBF) and the expression of trophic factors like. Recent research also reported chronic exercise promotes neurogenesis, angiogenesis and synaptogenesis [19] which are generally inhibited by aging, neurodegenerative diseases, accumulation of free radicals and stress [20]. Interestingly, van Praag [21] postulated that adhering to a balanced combination of voluntary exercise and a diet rich in antioxidants and anti-inflammatory compounds might have significantly better results than either diet or exercise alone [22].

Activation of the hypothalamus-pituitary-adrenal (HPA) axis, changed in accordance with the type, duration and intensity of physical exercise that an individual could perform. Physical exercise is an acute stressor, but interestingly, episodes of chronic exercises depicted neuroprotective effects. These effects were established by the finding that subjects who had undergone physical training had lower levels of cortisol both at rest and in response to a stressor than sedentary subjects [23]. Increased vasopressin/CRH ratio might have positive effects on this negative feedback for chronic stress through a reduction in pituitary stimulation [24].

Animal studies revealed that elevation of ROS concentration following exercise might activate the CREB protein and the peroxisome proliferator-activated receptor- $\gamma$ coactivator (PGC-1 $\alpha$ ) in the nucleus, thereby inducing mitochondrial biogenesis. Thus, chronic aerobic exercise may have both antioxidant-mediated and mitochondrial biogenic activity [25]. Chronic exercise can even result in changes in genetic structures, such as those of the telomeres in leukocytes. Individuals who practice moderate physical activity appear to have longer telomeres than sedentary 
individuals and those who practice exercise that is either higher or lower in intensity. The reported results regarding whether physical exercise enhances telomerase activity are still inconclusive [26].

\section{Adolescents' Gender Variability in Stress Response}

Eventually society in general and parents in particular have great expectations from their young members to become successful in all endeavours. Studies indicate those who cannot conform to their respective roles are looked down upon by their parents and society and as a consequence get stressed and isolated [27]. Studies on social psychology suggests that adolescent boys in general are more stressed because very rigid demands are placed on them regarding their career and expectation, as would be supporter of the family in future [28]. On the other hand, they are naturally restless owing to higher metabolic rate and cannot adjust to rigid school/college routine [29]. Another study in 100 students (50 males and 50 females) with the age ranged between 16-19 years from Mangalore used the Adolescents Stress Scale and semi structured interview and A Self report Coping Scale to report the patterns of stress, coping style and social support among the studied population. The results indicated that female students had greater stress scores related to college attendance, uncertainty about the future and the total score than males. It indicated a contradictory finding than the earlier cases. A separate study revealed that gender does not affect the stress response in adolescent learners [30]. However there was a significant difference between Tamil and English medium students and rural and urban students with respect to medium and location respectively [30].

\section{Psychological Backdrop of Adolescence Stress}

The psychological effect of stress is observed in terms of altered behaviour. Study revealed that people at adolescence face a number of psychological disturbances out of emotional stress due to self-identity crisis, inadequate selfconcept and variety of role expectations [31]. A study on 268 high school students revealed that life stress was found to be a significant predictor of depressed mood and poor cognitive performance [32].

There is considerable interest and controversy over the relatively new construct of emotion called, Emotional Intelligence (EI). Most people first heard the term with the publication of Daniel Goleman's best-selling book "Emotional Intelligence" [33]. EI comprises of four factors, e.g., selfawareness, self-management, awareness of others and relationship management. He stated EI is even more powerful determinant than IQ (Intelligence quotient) [33] for one's success in personal life and professional career as well. Hence, adolescents must exercise the means to develop it satisfactorily to meet the greater challenges in future course of time [34].
One study indicated the role of certain brain areas like Amygdala in expression of emotion [35]. Another study suggests a pathway for emotion that begins in the brain area where primary senses enter the brain [36]. The information then travel to the frontal area of brain in order to think rationally about what is experienced. However, the information must travel through the limbic system first and this is where emotions are generated [35].

Emotional intelligence (EI) refers to a set of competencies that are essential features of human social life. Although the neural substrates of EI are virtually unknown, it is well established that the prefrontal cortex (PFC) plays a crucial role in human social-emotional behaviour [37]. A study was done on veterans subjects with focal penetrating head injuries. The Mayer-Salovey-Caruso Emotional Intelligence Test was applied on them. The results revealed that key competencies underlying EI depend on distinct neural PFC substrates. First, ventromedial PFC damage diminishes Strategic EI, and therefore, hinders the understanding and managing of emotional information. Second, dorsolateral PFC damage diminishes Experiential EI, and therefore, hinders the perception and integration of emotional information. In conclusion, EI should be viewed as complementary to cognitive intelligence and, when considered together, provide a more complete understanding of human intelligen [38].

Stress Coping Strategy in adolescence: Review of existing literature suggests, exercise can play pivotal role in physiological coping of stress while development of emotional intelligence plays significant role in psychological adaptation to stress in adolescence [39]. One recent study from human brain imaging and animal studies showed strong inclination to incentives and socioemotional contexts during adolescence though the impulse control is still relatively immature at this time. These findings suggest involvement of limbic systems for emotional processing [40]. Another work revealed significant interactions between physical exercise and emotion on both the genders [41]. The girls were reported to less favourably assessed their health, experienced more somatic complaints and manifested more marked negative mood states than their male counterparts.

\section{Coping Strategies of Adolescence Stress}

The current study is focused on the psychological and physiological ways of managing stress in adolescents. More precisely the psychological way involving emotional intelligence skill and exercise mediated physiological means of coping strategies have been discussed here.

\subsection{Psychological Coping (EI Mediated) of Adolescence Stress}

Researchers have claimed with little empirical support that emotional intelligence may be the best predictor of success in all spheres of life, whether in relationships, competition, 
academics [42]. As emotional intelligence is a subset of social intelligence with the ability to understand and monitor one's own feelings and others too. So adolescents require adequate EI to ensure social adjustment [43].

One related study aimed to enumerate the factors responsible to affect the development of emotional intelligence and its role in academic stress for adolescents [43]. In this research secondary data was collected out of which the correlation between EI and academic stress was analysed. The finding showed emotional skills at school not only positively influence academic achievement during the adolescence year when these were taught but also leaves the impact in long term achievement by overcoming stress [44].

Findings of another work hypothesised that academic achievement without EI does not indicate future success and absence of EI also indicated the weak personality and ability to cope up with moderate levels of stress [45].

Collaborative research between Swinburne University and a number of schools from Australia studied how adolescent EI is related to a range of important educational variables including scholastic success; problem behaviours (externalised and internalised); and stress coping strategies. It was depicted that low EI were more likely to engage in aggressive and rule-breaking behaviours and got stressed easily [46]. They were more likely to experience symptoms of anxiety, depression, withdrawal, attention, social problems and low academic achievement [46]. Emotional regulation of the self was positively correlated with plan full problem solving, confronting coping, self-controlling and positive reappraisal, but negatively correlated with escape avoidance [47]. No gender difference was reported in development of emotional intelligence and ways of coping stress except for self-control, where males reported higher capability than females [48]. Emotional trauma contributed greatly to disease and it has been reflected by Emotional Freedom Technique (EFT) that combines a cognitive and a somatic element. EFT is able to rapidly reduce the emotional impact of memories and incidents that triggered emotional distress. Once the distress is reduced or removed, the body could often rebalance itself, and accelerated healing. The mechanism is that, EFT first elicits the midbrain's anxiety reflex and then replaces it with a relaxation response; in order to - reciprocally inhibit anxiety. It also indicated that manual stimulation of acupuncture points produced opioids, serotonin, and gamma-aminobutyric acid (GABA), and thus regulated the stress hormone cortisol. These neurochemical changes lead to reduction of pain, slowing of the heart rate, decreased anxiety, shutting off the fight of flight response, and regulation of the autonomic nervous system, as well as to the creation of a sense of calm. Therefore application of EFT is beneficial to reduced pain perception, increased acceptance, coping ability and health-related quality of life in individuals although there was no gender variation found in this context [49].

\subsection{Physiological (Exercise Mediated) Coping of Stress}

Physical exercise is an important tool for physiological coping of stress [50]. Group jogging exercise may be effective in improving depressive state, hormonal response to stress and physiological fitness of adolescent females with depressive symptoms [48]. A prospective work on health and exercise was made to investigate stress and well-being in adolescence and to evaluate the ability of exercise to buffer stress-induced deteriorations in physical health. 384 females of 8th grade completed a questionnaire on day-to-day life events, the Seriousness of Illness Rating Scale and an activity questionnaire at the beginning and end of the academic year [51]. Analysis revealed that the negative impact of stressful life events on health declined as exercise levels increased. Findings also revealed that exercise might be a valuable resource for combating life stress.

The circulatory and thermal adjustment mechanism during heat stress has been well documented long back in adults [52]. Relatively recent work indicated adolescencents due to their rapid physiological changes are less efficient to adjust heat stress. It is well documented that low intensity endurance performance are very effective in initial stage of heat acclimatization in adolescents [53].

A related work suggests low-income adolescent individuals are more prone to stress than high-income individuals [54]. Low-income participants did report experiencing more stress than there high-income counterparts. So exercise could be more effective strategy for low-income group as their requirement is more than the other group [54].

The physiological effect of stress exposure induces a decrease in BDNF mRNA levels which can lead to depression [55]. Exercise is reported as a measure of treatment or prevention for stress-induced decrease in BDNF expression in brain associated with acute stress exposure. Regular physical activity increases cerebral blood flow, changes hormone levels, enhances nutrient intake, increases energy and improves concentration of mind [56]. Researches revealed that exercise in almost any form can act as a stress reliever [56].To combat these detrimental effects on the body, exercise is a natural way of getting rid of everyday stress.

\subsection{Exercise-Emotion Interface in Coping Adolescence Stress}

Positive impact of exercise on improving EI by some physiological mechanisms has been hypothesised in various studies.

Adolescent populations were subjected to a randomized controlled trial to investigate the effects of physical exercise on depressive state of mind. After the sessions of exercise the Centre for Epidemiologic Studies Depression (CES-D) scale, were randomly assigned to either an exercise regimen or usual daily activities for 8 weeks [57]. Twenty-four hour excretions of cortisol and epinephrine in urine were reduced due to the exercise regimen. The training group had a significantly reduced resting heart rate and increased peak oxygen uptake and lung capacity [57]. Regular exercise increased the self-confidence and lowered the symptoms associated with mild depression and anxiety 
[58]. Exercise also improved sleep, which is often disrupted by stress, depression and anxiety [58]. Some researchers reported remarkable benefits of regular exercise on developing EI [58]. Another study suggested exercise mediated release of feel-good endorphins keeps the individual distracted from stress of day-to-day life and boosts him to remain active mentally [59].

The above discussion leads to the final interpretation that the various aspects of psycho-physiological development in adolescence like physical growth, cognitive development, emotional and behavioural maturation etc. get retarded at this stage by over stress. There are two predominant ways of coping stress for adolescents namely, psychological approach and physiological approach Development of EI seems very effective means of psychological coping, while exercise deserves special mention for Physiological mechanism of stress coping. Additionally, exercise contributes significantly on developing EI in adolescents.

Exercise was reported to improve attention, reading comprehension in adolescents with socio-economic background, who normally pass more through stressful life situations than their high-income counter parts.

In psychological approach, adolescents having high EI can rapidly cope with stress by relaxation and meditation techniques. Progressive muscle relaxation can reduce physical tension and meditation can reduce stress and anxiety.

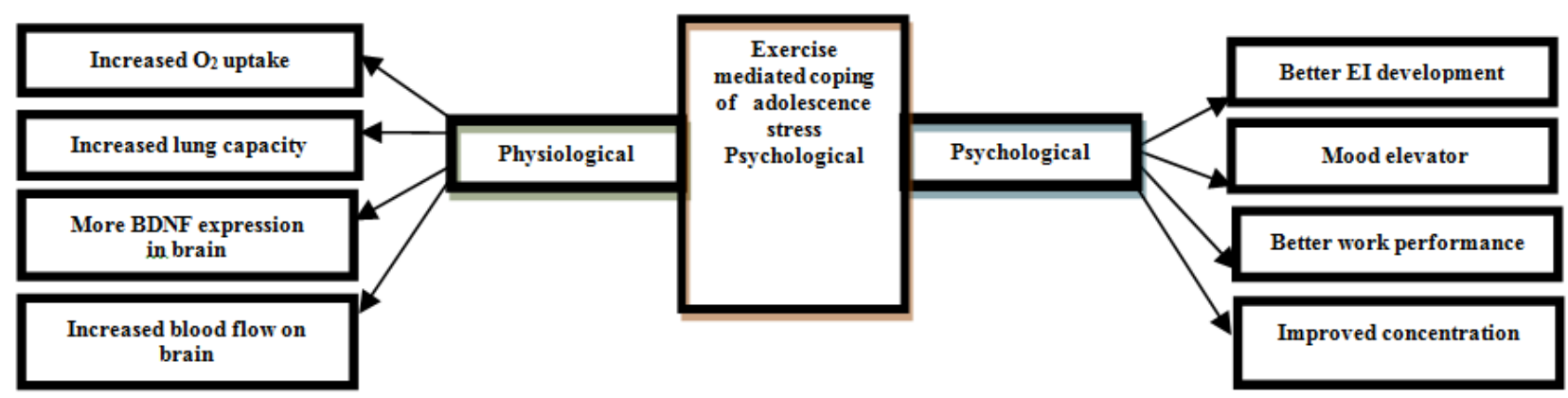

Figure 1. Schematic presentation of exercise-emotion interface in coping adolescence stress.

\section{Conclusions}

The current review revealed that considerable works have been carried out so far in addressing adolescence stress from physiological and psychological perspectives. In this regard the role of exercise and emotional intelligence in combating stress has been well established. However, till date literature seems inconclusive to confirm adolescent boys are more prone to stress than their female counterparts or vice versa. Again, the development of EI is suggested to be a powerful mediator for coping stress in adolescents. Though, circumstantial evidence is inadequate to prescribe a standard neurological mechanism of its action in managing stress. Moreover, research areas like family studies, genetics, and neurobiology are not touched adequately. In India research related to Neurophysiological and Neuropsychological basis of adolescence is dismally low. Furthermore research is lacking in the areas of non-pharmacological management like relaxation therapies, yoga, and other meditation techniques to manage stress in adolescents in India. This review clearly emphasized that physical exercise is a strong weapon to build the physical and mental capabilities in adolescent populations. This early young generation must be encouraged from all levels to take part in regular physical exercise programme and practising EI skills to develop a healthy personality that in turn would help to develop a healthy society. Thrust should be imposed from concerned levels to introduce and frame physical education programme as a mandatory part of the School education system. Moreover, this literature will be helpful to prescribe proper physical training guideline and relaxation techniques to build their ability to cope with psychosocial stress. If required, necessary counselling methods also need to be employed to strengthen the psychological ability to sustain physical and mental stress in this group of populations.

\section{References}

[1] M. H. Anshel, and D.Q. Marisi, Effect of music and rhythm on physical performance. Research Quarterly, 1978, 49: 109113.

[2] P. R. Huli, Stress Management in Adolescence. Journal of Research in Humanities and Social Sciences, 2014, 2: 50-57.

[3] I. Ajzen, and M. Fishbein, Factors influencing intentions and the intention- behaviour relation. Human Relations, 1974, 27: $1-15$.

[4] M.H. Anshel, and M. Kang, An outcome-based action study on changes in fitness, blood lipids, and exercise adherence based on the disconnected values (interventional) model. Behavioural Medicine, 2007, 33: 85-100.

[5] B.G. Berger, and R. Motl, Exercise and mood: A selective review and synthesis of research employing the profile of mood states. Journal of Applied Sport Psychology, 2000, 12: 69-92.

[6] R.C. Eklund, and S. Crawford, Active women, social physique anxiety and exercise. Journal of Sport and Exercise Psychology, 1994, 16: 431-448. 
[7] W.A. Fisher, J.D. Fisher, and J.J. Harmon, The informationmotivation - behavioural skills model as a general model of health behaviour change: Theoretical approaches to individual-level change. In J. Suls \& K. Wallston (Eds.), Social psychological foundations of health, 2003, London: Blackwell, pp. 127-153.

[8] H. A. Hausenblas, A.V. Carron, and D.E. Mack, Application of the theories of reasoned action and planned behaviour to exercise behaviour: A meta-analysis. Journal of Sport and Exercise Psychology, 1997, 19: 36-51.

[9] H.A. Hausenblaus, and D.A. Symons, How much is too much? The development and validation of the Exercise Dependence Scale. Psychology and Health, 2002, 16: 387404.

[10] E. Diener, Subjective well-being: The science of happiness and a proposal for a national index. American Psychologist, 2000, 55: 34-43.

[11] C. I. Karageorghis, and P. C. Terry, The psychophysical effects of music in sport and exercise: A review. Journal of Sport Behaviour, 1997, 20: 54-68.

[12] P.D. Tomporowski, Exercise and children's Intelligence, Cognition and academic achievement. Journal of Education and Psychology Review, 2008, 20: 111-131.

[13] D.S. Kirschenbaum, Self-regulation and sport performance. Medicine and Science in Sports and Exercise, 1987, 19: 106113.

[14] E.M.M. Portugal, T, Cevada, S.R. Monteiro, G.T. Teixeira, R.E. da-Cruz, E. Lattari E et al., Neuroscience of Exercise: From Neurobiology Mechanisms to Mental Health. Neuropsychobiology, 2013, 68: 1-14.

[15] A. Deslandes, H. Moraes, C. Ferreira, H. Veiga, H. Silveira, R. Mouta, F.A. Pompeu, and E.S. Coutinho, Exercise and mental health: many reasons to move. Neuropsychobiology, 2009, 59: 191-198.

[16] R.K. Dishman, H.R. Berthoud, F.W. Booth, C.W. Cotman, V.R. Edgerton, and M.R. Fleshner, Neurobiology of Exercise. 2006, 14: 345-356.

[17] S.N. Sarbadhikari, and A.K. Saha, Moderate exercise and chronic stress produce counteractive effects on different areas of the brain by acting through various neurotransmitter receptor subtypes: a hypothesis. Theory of Biological Medicinal Model, 2006, 3: 33-39.

[18] A. Dietrich, and W.F. McDaniel, Endocannabinoids and exercise. Br J Sports Med, 2004, 38: 536-541.

[19] R.S. Duman, Neurotrophic factors and regulation of mood: role of exercise, diet and metabolism. Neurobiology of Aging, 2005, 26: 88-93.

[20] A.A. Walf, J.J. Paris, M.E. Rhodes, J.W. Simpkins, and C.A. Frye, Divergent mechanisms for trophic actions of estrogens in the brain and peripheral tissues. Brain Research, 2011, 1379: 119-136.

[21] H. Van Praag, Exercise and the brain: something to chew on. Trends in Neuroscience, 2009, 32: 283-290.

[22] A.C. Pereira, D.E. Huddleston, A.M. Brickman, A.A. Sosunov, R. Hen, G.M. McKhann, R. Sloan, F.H. Gage, T.R. Brown, and S.A. Small, An in vivo correlate of exercise- induced neurogenesis in the adult dentate gyrus. Proceeding of National Academy of Science USA, 2007, 104: 56385643 .

[23] E. Papacosta, and G.P. Nassis, Saliva as a tool for monitoring steroid, peptide and immune markers in sport and exercise science. Journal of Science and Medicine in Sport, 2011, 14: 424-434.

[24] S.L. Lightman, The neuroendocrinology of stress: a neverending story. Journal of Neuroendocrinology, 2008, 20: 880884 .

[25] S.K. Powers, E.E. Talbert, and P.J. Adhihetty, Reactive oxygen and nitrogen species as intracellular signals in skeletal muscle. Journal of Physiology, 2011, 589: 2129-2138.

[26] R.J. Simpson, and K.Guy, Coupling aging immunity with a sedentary lifestyle: has the damage already been done? A mini-review. Gerontology, 2010, 56: 449-458.

[27] E. McAuley, E, and S.L. Mihalko, Measuring exercise-related self-efficacy. In J. L. Duda (Ed.), Advances in sport and exercise psychology measurement, Morgantown, WV, 1998: Fitness Information Technology, pp. 371-390.

[28] B. H. Marcus, B.C. Bock, B.M. Pinto and M.M. Clark, Exercise initiation, adoption, and maintenance. In J. L. Van Raalte \& B. W., Brewer (Eds.), 1996, 1,133-159.

[29] J.K. Trivedi, An overview of Indian Research in Anxiety Disorders. Indian Journal of Psychiatry, 2010, 52: 210-218.

[30] K. Manikandan, and S. Nirmala Devi, A study on stress among adolescent learners. Scholarly Research Journal for Interdisciplinary studies, 2015, 2: 2725-2730.

[31] M.J. Priester, and G.A. Clum, The problem-solving diathesis in depression, hopelessness, and suicide ideation: a longitudinal analysis. Journal of Psychopathology and Behavioural Assessment, 1993 15, 239-254.

[32] N. Schutte, J. Malouff, L. Hall, D. Haggerty, J. Cooper, C. Golden, and L. Dornheim, Development and validation of a measure of emotional intelligence. J. Personality and Individual Differences, 1998, 25: 167-177.

[33] J. D. Mayer, D. Caruso, and P. Salovey, Emotional intelligence meets traditional standards for an intelligence. Intelligence, 1999, 27: 267-298.

[34] J. D. Mayer, and G. Geher, Emotional intelligence and the identification of emotion. Intelligence, 1996, 22: 89-113.

[35] J.C. Tarasuik, J. Ciorciari, and C. Stough, Understanding the Neurobiology of Emotional Intelligence: A Review,The Springer Series on Human Exceptionality. 2009 pp 307-320.

[36] R.H. Prerana, Stress Management in Adolescence. Journal of Research in Humanities and Social Science, 2014, 2: 50-57.

[37] K.R. Murphy, and C.O. Davidshofer, Psychological testing: principles and applications (4th ed.). 1998, New Jersey: Prentice-Hall, Inc., pp. 201-207.

[38] J. Nemiah, H. Freyberger, and P.E. Sifneos, Alexithymia: a view of the psychosomatic process. In O. W. Hill, London: Butterworths.1976, Vol. 3, pp. 430-439.

[39] E. Diener, Subjective well-being: The science of happiness and a proposal for a national index. American Psychologist, 2000, 55: 34-43. 
[40] B.J. Casey, R.M. Jones, T.A. Hare, The Adolescent Brain. Annals of the New York Academy of Sciences, 2008, 1124:111-126.

[41] N.H. Miller, M. Hill, T. Kottke, and I. S. Ockene, The multilevel compliance challenge: Recommendations for a call to action. Circulation, 1997, 95: 1085-1090.

[42] J. Simpson, W. Ickes, and T. Blackstone, When the head protects the heart: Empathic accuracy in dating relationships. Journal of Personality and Social Psychology, 1995, 69: 629641.

[43] Goleman D. (1998). Working with Emotional Intelligence, NewYork: Bantam Books.

[44] M.J. Elias, M. Gara, T. Schuyler, L.R. Brandon-Muller, and M.A. Sayette, The promotion of social competence: longitudinal study of a preventive school-based programme. American Journal of Orthopsychiatry, 1991, 61: 409-417.

[45] K.V. Petrides, N. Frederickson, and A. Furnham, The role of trait emotional intelligence in academic performance and deviant behaviour at school. Journal Personality and Individual Differences, 2004, 36: 277-293.

[46] J.D.A. Parker, L.J. Summerfeldt, M.J. Hogan, and S. Majeski, Emotional Intelligence and academic success: Examining the transition from high school to university. Journal of Personality and Individual Differences, 2004, 36: 163-172.

[47] L. Varvogli, Stress Management Techniques: evidence-based procedures that reduce stress and promote health. Health Science Journal, 2011, 74: 74-89.

[48] R. Rubin, and W. Bommer, Emotional intelligence and individual performance: Evidence of direct moderated effects. Journal of Organizational Behaviour, 2007, 28: 399-421.

[49] P.J.V. Beumont, I.S. Fraser, and D. Llewellyn-Jones, Body weight, exercise and menstrual status among ballet dancers in training. British Journal of Obstetrics and Gynaecology, 1982, 89: $507-510$

[50] L.K. Bachrach, D.K. Katzman, D. Guido, and R. Marcus. Recovery from osteopenia in adolescent girls with anorexia nervosa. Journal of Clinical Endocrinology and Metabolism, 2007, 72: 602-606.

[51] C.S. Brokenshire, The Reliability of Adolescent Thermoregulatory Responses During a Heat Acclimation Protocol. Journal of Sports Science and Medicine, 2009, 8: 689-695.

[52] B. Falk, R. Dotan, Children's thermoregulation during exercise in the heat - a revisit. Journal of Applied Physiology Nutrition and Metabolism, 2008, 33: 420-427.

[53] A. Ballau, A. Ziegler, A. Emons, G. Sturm, and W.F. Blum, H. Remschmidt and J. Hebebrand, Serum leptin and gonadotropin levels in patients with anorexia nervosa during weight gain. Molecular Psychiatry, 1999, 4: 71-75.

[54] A. Bonen, A.N. Belcastro, W.Y. Ling, and A.A. Simpson, Profiles of selected hormones during menstrual cycles of teenage athletes. Journal of Applied Physiology, 1981, 50: 545-551.

[55] M.M. Eduardo, Neuroscience of exercise. Journal of Neuropsychobiology, 2013, 68: 1-14.

[56] R. S. Paffenbarger, R.T. Hyde, A.L. Wing, I.M. Lee, D.L. Jung, and J.B. Kampert, The association of changes in physical-activity level and other lifestyle characteristics with mortality among men. New England, 1993, 328: 538-545.

[57] N.E. Adler, M. Marmot, B.S. McEwen, and J.E. Stewart, Socioeconomic Status and Health in Baliki Industrial Nations: Social, Psychological, Biological Pathways. New York Academy of Sciences. 1999.

[58] C.C. Cheung, J.E. Thornton, J.L. Kuijper, D.S. Weigle, D.K. Clifton, and R.A. Steiner, Leptin is a metabolic gate for the onset of puberty in the female rat. Endocrinology, 1997, 138: 855-858.

[59] K. Clement, C. Vaisse, N. Lahlou, S. Cabrol, V. Pelloux, D. Cassuto, M. Gourmelen, and C. Dina, A mutation in the human leptin receptor gene causes obesity and pituitary dysfunction. Nature, 1998, 392: 398-401. 